\title{
Effect of laser energy and repetition rate on holmium plasma
}

\section{emission}

\author{
Nibras N. mahmood and Mahmoad SH. Mahmoad \\ Institute of Laser for Postgraduate Studies, University of Baghdad, Baghdad, Iraq \\ E-mail: Niprasnazeh@yahoo.com
}

\begin{abstract}
The plasma holmium produced by a $1064 \mathrm{~nm}$ Nd:YAG Q-switched laser in air was investigated. This work was done theoretically and experimentally. Cowan code was used to get the emission spectra for different transition of the holmium target. In the experimental work, the plasma evolution has been studied by acquiring spectral images with different laser pulse energies (600, $650,700,750$, and 800$) \mathrm{mJ}$, with The repetition rates of $(1 \mathrm{~Hz}$ and $10 \mathrm{~Hz}$ ). In the UV region (200-400) nm. The results indicate that, the emission line intensities increase with increasing of the laser pulse energy and repetition rate. The strongest emission spectra appeared when the laser pulse energy is $800 \mathrm{~mJ}$ and $10 \mathrm{~Hz}$ repetition rate at $\lambda=345.64 \mathrm{~nm}$, with the maximum intensity of 77000 counts.
\end{abstract}

Key words

Emission spectra, pulse energy, $\mathrm{Nd}$ -

YAG laser, holmium.

Article info.

Received: Dec. 2019

Accepted: Jan. 2020

Published: Mar. 2020

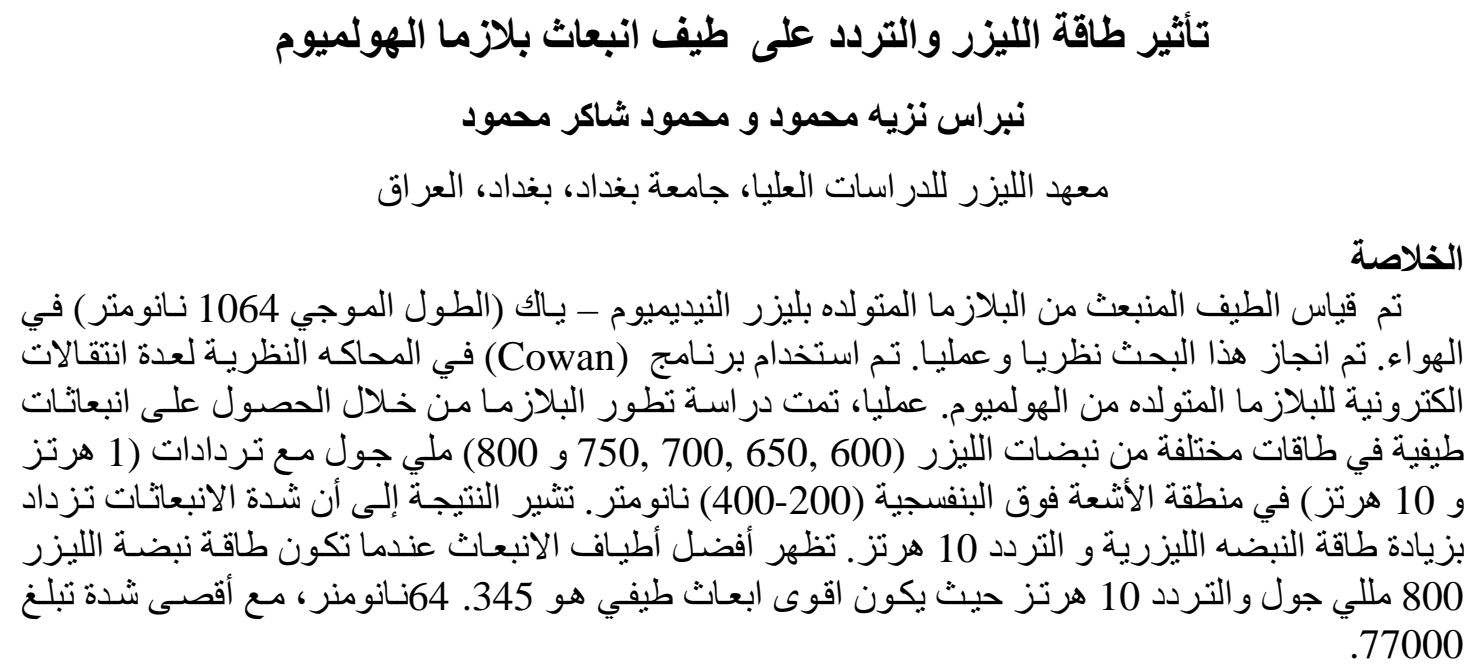

\section{Introduction}

The plasma produced by intense laser pulse can give information about qualitative and quantitative analyses of the sample [1]. The emission spectra of Plasma provide spectral signatures of many different type of materials in solid, liquid, and gas state [2]. The plasma emitted light consists of discrete lines, bands, and an overlying continuum [3]. The continuum plasma emission are emitted as a result of free- free and free-bound transitions. It is very important to determine the parameter of plasma, such as electron temperature, and density of electron, which is very important to characterize plasma [4, 5]. Transitions between bound states in an atom give rise to discrete lines. Such lines observed in both absorption and emission spectra. These discrete lines is very important. to characterize the material, and have three main features. Wave length, 
intensity, and shape [6, 7]. The parameter depend on both the structure of the emitting atoms, and environment. In this work, pure holmium ( $\mathrm{Z}=67$ ) was used, Holmium (Ho) are lanthanides, the eleventh element [7], or the rare Earth group element. That has one stable isotope (165Ho). This isotope has a nuclear spin of $I=7 / 2$. [8, 9]. The rare earth Spectra, are of astrophysical interest, for example, the experiment data is needed for quantitative evaluation of abundances element, because of $4 \mathrm{f}$ electron shell is unfilled [10]. The spectra of all rare earth elements are very dense and complex [11]. All this pectra are far from being fully identified [12]. In general," more than half of the lines that occur in a rare earth spectrum are neither currently classified nor classifiable on the basis of known energy levels" [13, 14]. The Cowan code is the most widely available numerical computer code for calculating atomic structure. This FORTRAN code was comprised by
Robert D. Cowan in 1968 [15]. This code was originally written in 1961, and has been developed over a long period by Cowan and co-workers since 1964 [16]. Based on Hartree-Fock equations, it also employs several other approximations such as Hartree-Fock with exchange in which different methods are used for self-interaction correction and another method to approximate the remainder of the Hartree-Fock exchange term [17].

The main focus of this paper is to classify the spectral lines intensity of holmium target in the (200-400) nm spectral range at different laser pulse energy with different repetition rate. The results obtained showed that the emission intensity changes considerably with the laser energy used.

\section{The experimental set-up}

The experimental setup for obtaining the emission spectra for the plasma in the uv region can be shown in Fig.1.

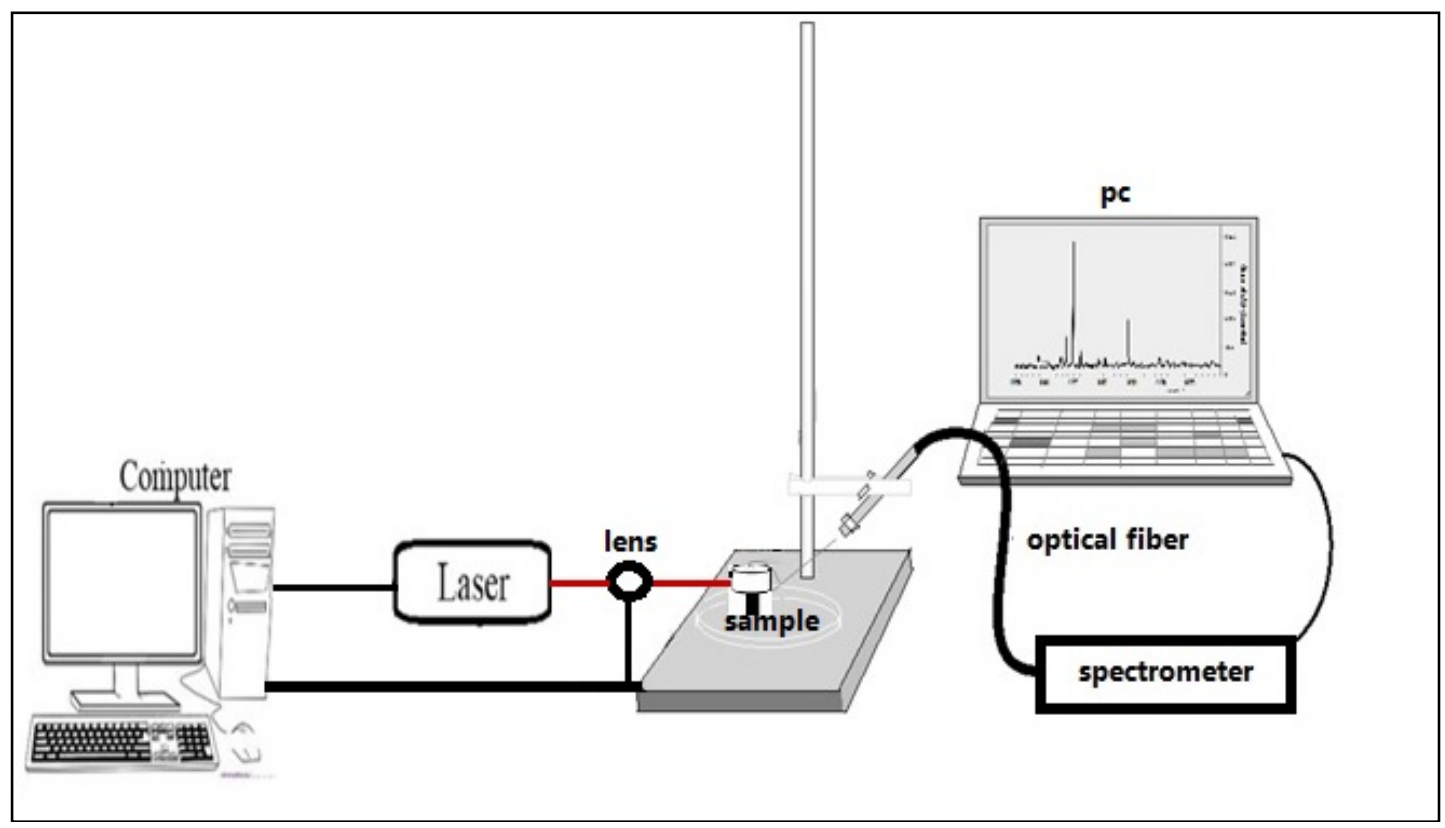

Fig.1: Schematic diagram of the experimental setup for laser induced plasma.

The setup consist of laser Nd:YAG source for plasma produced and vaporization of the sample. The 1064nm beam of Nd:YAG Q-switched laser. Pulse width (10 ns). 1-20 Hz pulse repetition rate, emitting a laser 
pulse with maximum energies $900 \mathrm{~mJ}$. The laser are focused horizontally on the target of holmium surface by Biconvex lens with $100 \mathrm{~mm}$ focal length. Distance between the lens to the holmium are about $100 \mathrm{~mm}$ in order to reproducible breakdown, and to get a nearly spherical plasma plume. The plasma produced in air at atmospheric pressure on $1.75 \mathrm{~mm}^{2}$ surface area of the sample. $99.9 \%$ holmium purity content. The emission are collected by the optical fiber which was set at angle of about 45 degree of the target and diverted into a spectrometer connected to the laptop. According to the specifications of this spectrometer, the resolution is $0.1 \mathrm{~nm}$ ( 1 angstrom). are a fiber optically coupled instruments with selection of measurements in the wavelength range (200-400) $\mathrm{nm}$, has a high resolution to analyze the emission lines spectra. Different laser parameters have been used in this work. Different laser pulse energies of (600, 650, 700, 750, and 800) $\mathrm{mJ}$ are focused each time with different repetition rate of $(1 \mathrm{~Hz}$, and $10 \mathrm{~Hz})$.

\section{Results \& dicussion}

1- Holmium Emission at $1 \mathrm{~Hz}$ repetition rate

The maximum intensity of holmium line emission was at $(\lambda=345.75 \mathrm{~nm})$, when the laser pulse energy is $600 \mathrm{~mJ}$ with a maximum intensity of 40000 , as shown in Fig.2.

Fig.3 shows the emission spectra result when the laser pulse energy is $650 \mathrm{~mJ}$. The result obtained from these parameter shows many line emission spectra of holmium, and the strongest one is $345.75 \mathrm{~nm}$ with a maximum intensity of 59000 .

When the laser energy was increased to $700 \mathrm{~mJ}$, the intensity of the emission line is also increased, blue shift has been observed whereas the maximum intensity of emission spectra was (65535 at $\lambda=341.43 \mathrm{~nm}$ ), as shown in the Fig.4.

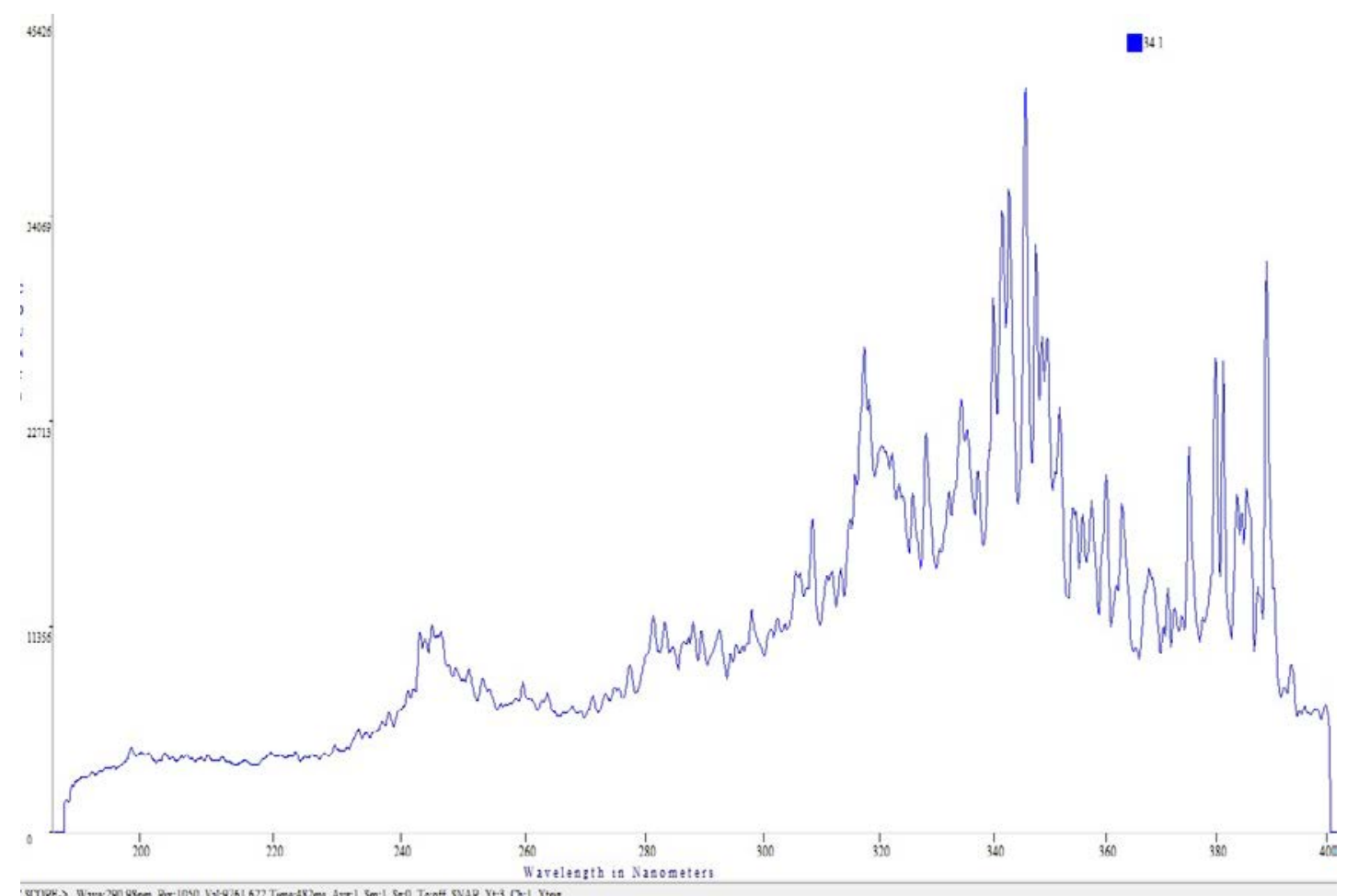

Fig. 2: Emission spectra of laser energy $600 \mathrm{~mJ}$ with $1 \mathrm{~Hz}$ repetition rate. 


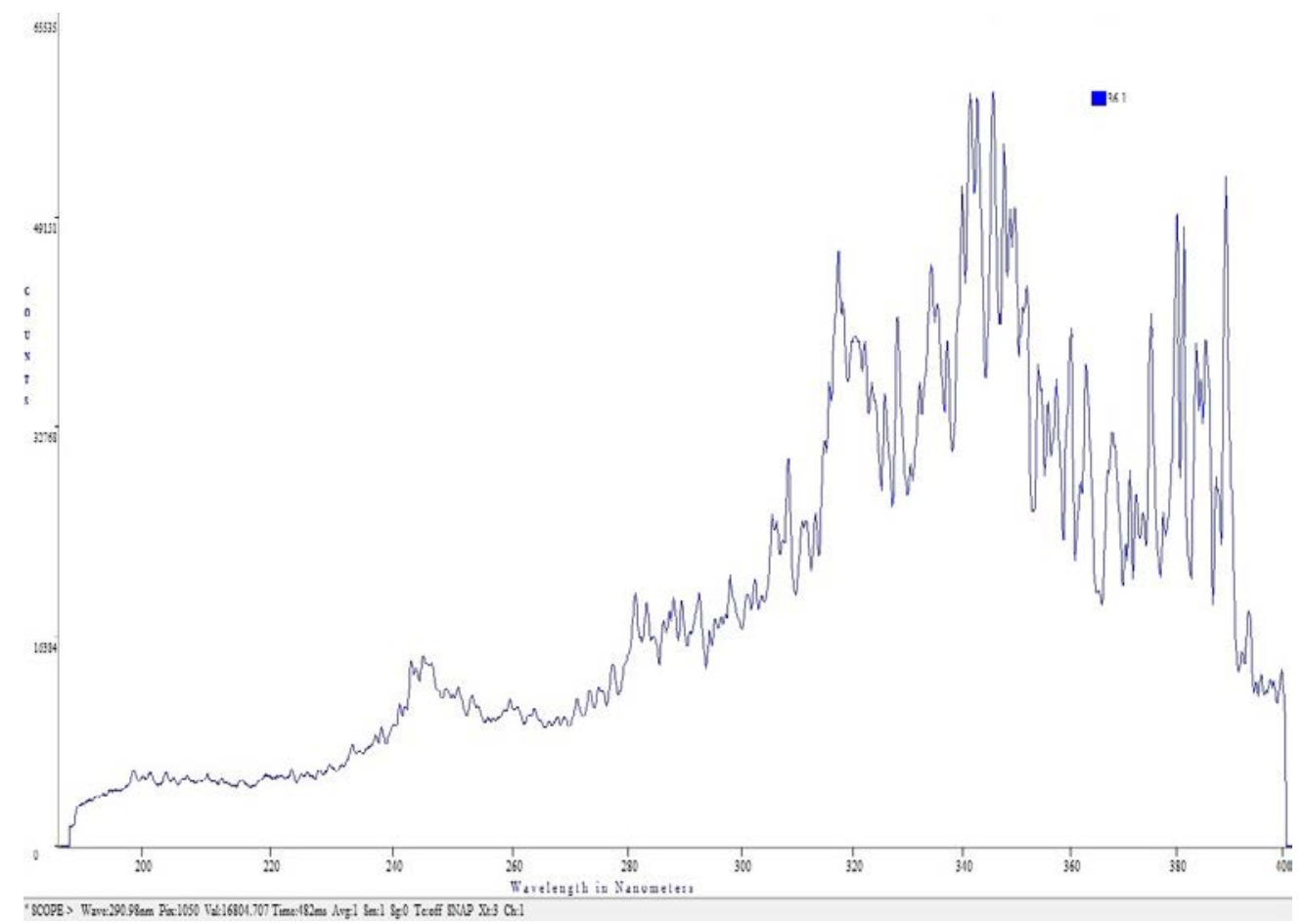

Fig.3: Emission spectra of laser energy $650 \mathrm{~mJ}$ with (1Hz) repetition rate.

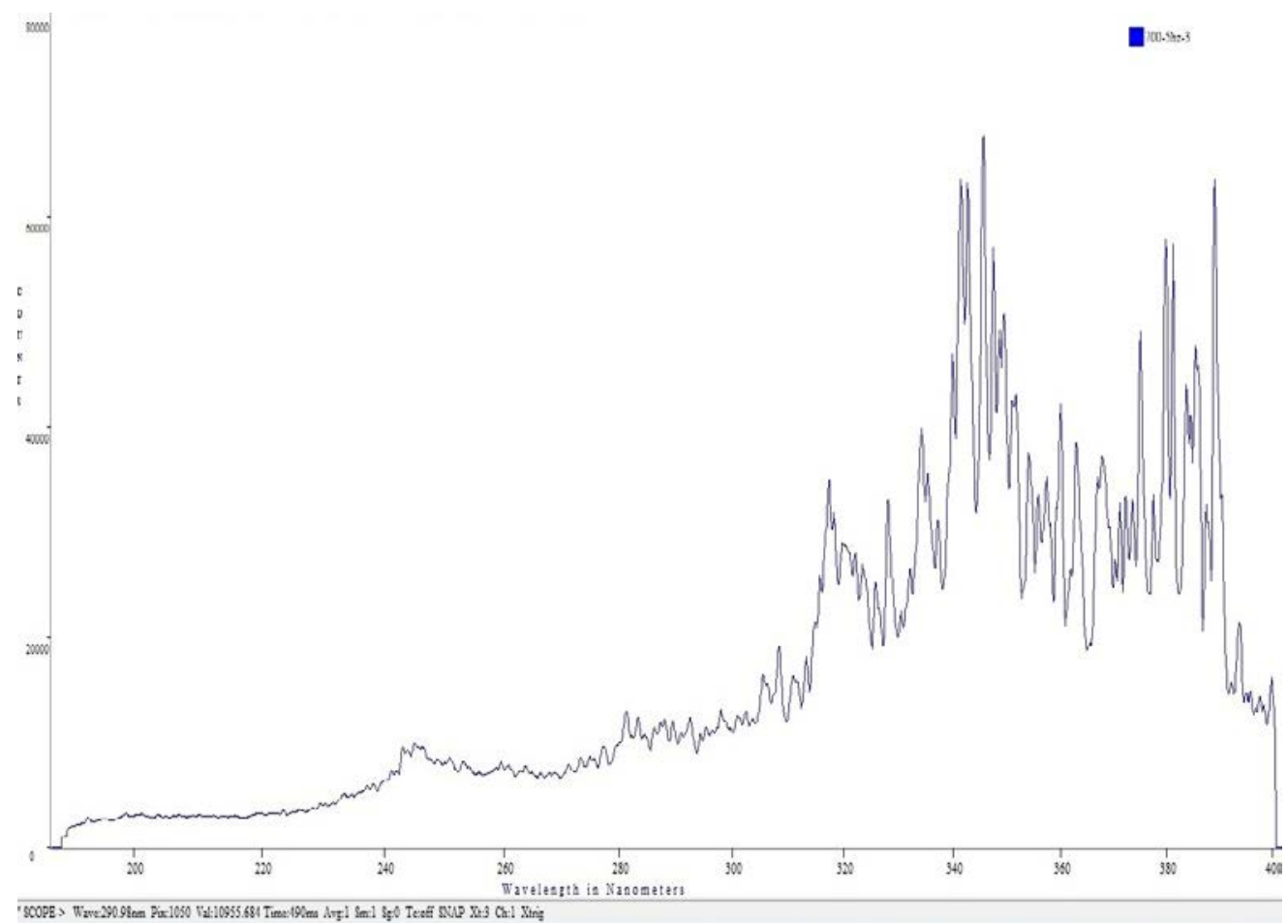

Fig.4: Emission spectra of laser energy $700 \mathrm{~mJ}$ with $(1 \mathrm{~Hz})$ repetition rate.

If the laser pulse energy is increased to $750 \mathrm{~mJ}$, the intensity of the emission line is also increased, and the maximum intensity of emission spectra was (70000 at $\lambda=341.54 \mathrm{~nm})$, as shown in the Fig.5.
As it shown in Fig.6, when the laser pulse energy is increased to $800 \mathrm{~mJ}$, the maximum intensity of the emission spectra was (80000 at $\lambda=341.54(\mathrm{~nm})$ ). 


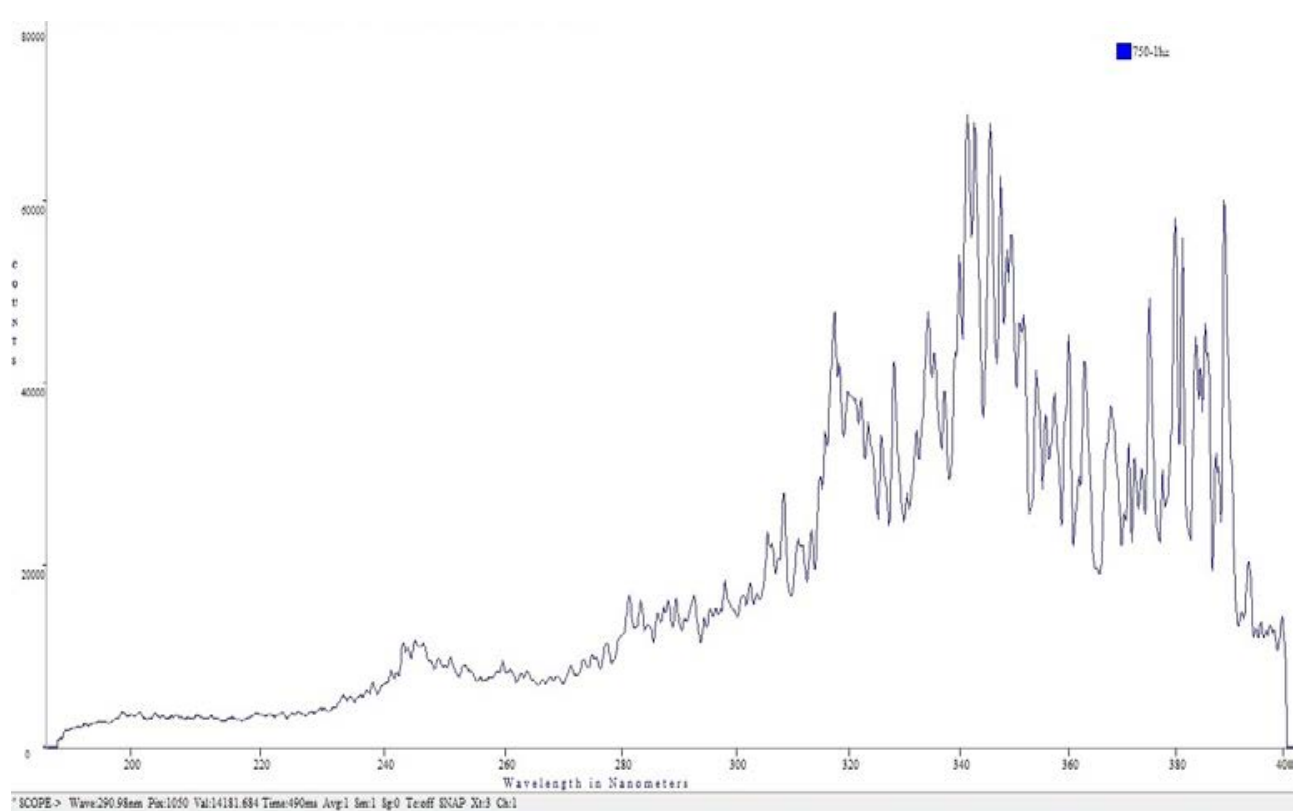

Fig.5: Emission spectra of laser energy $750 \mathrm{~mJ}$ with $(1 \mathrm{~Hz})$ repetition rate.

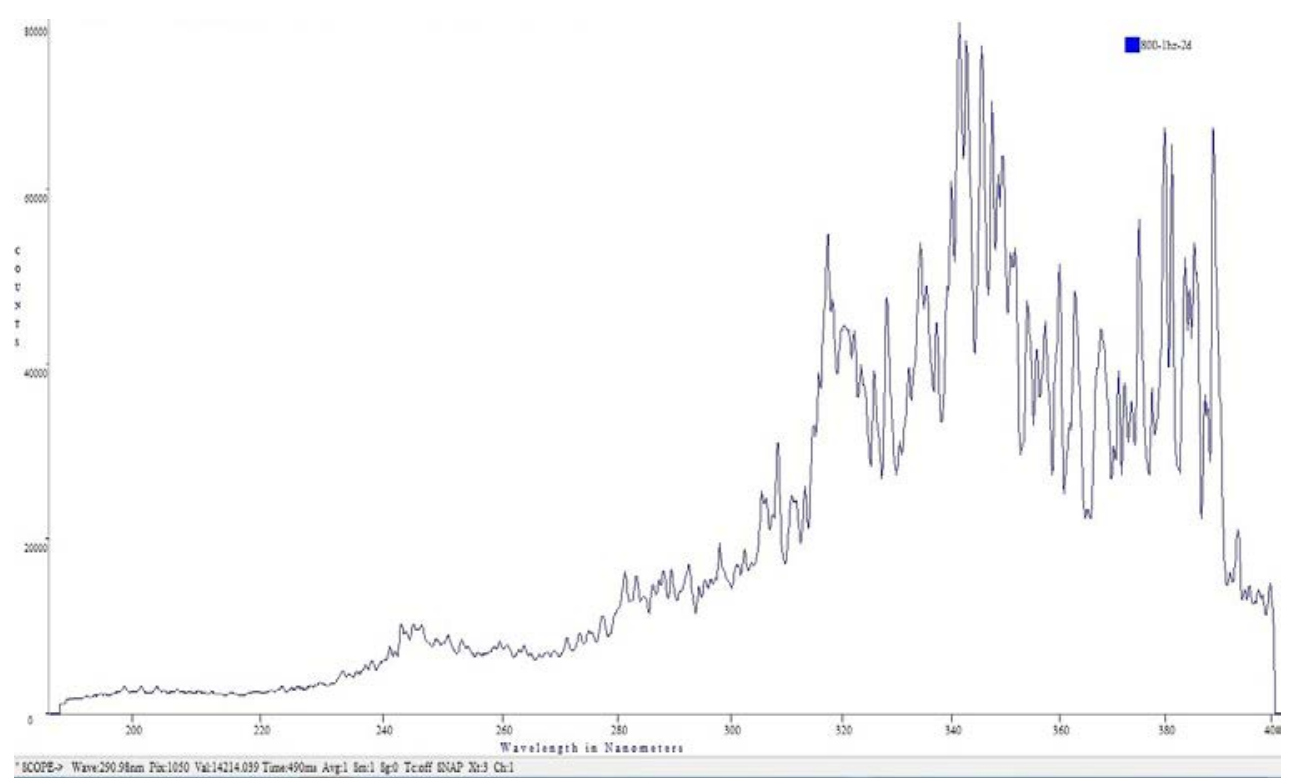

Fig.6: Emission spectra of laser energy $800 \mathrm{~mJ}$ with $(1 \mathrm{~Hz})$ repetition rate.

Fig.7 represents all the result for different laser pulse energy used in this work and the maximum holmium line emission intensity was ((80000 at $x=$ $341.54 \mathrm{~nm})$.

\section{2- Holmium emission at $10 \mathrm{~Hz}$ repetition rate}

When the laser pulse energy was $600 \mathrm{~mJ}$, the maximum intensity of holmium line emission was at $(\lambda=345.75 \mathrm{~nm})$, with the maximum intensity of (40000), as shown in Fig.8.
Fig.9 shows the emission spectra result when the laser pulse energy is $650 \mathrm{~mJ}$. The result obtained from these parameter shows many line emission spectra of holmium, and the strongest one is $345.53 \mathrm{~nm}$ with a maximum intensity of 45000 . When the laser energy was increased to $700 \mathrm{~mJ}$, the intensity of the emission line is also increased, and the maximum intensity of emission spectra was (51070 at $\lambda=345.53 \mathrm{~nm}$ ), as shown in the Fig. 10 . 


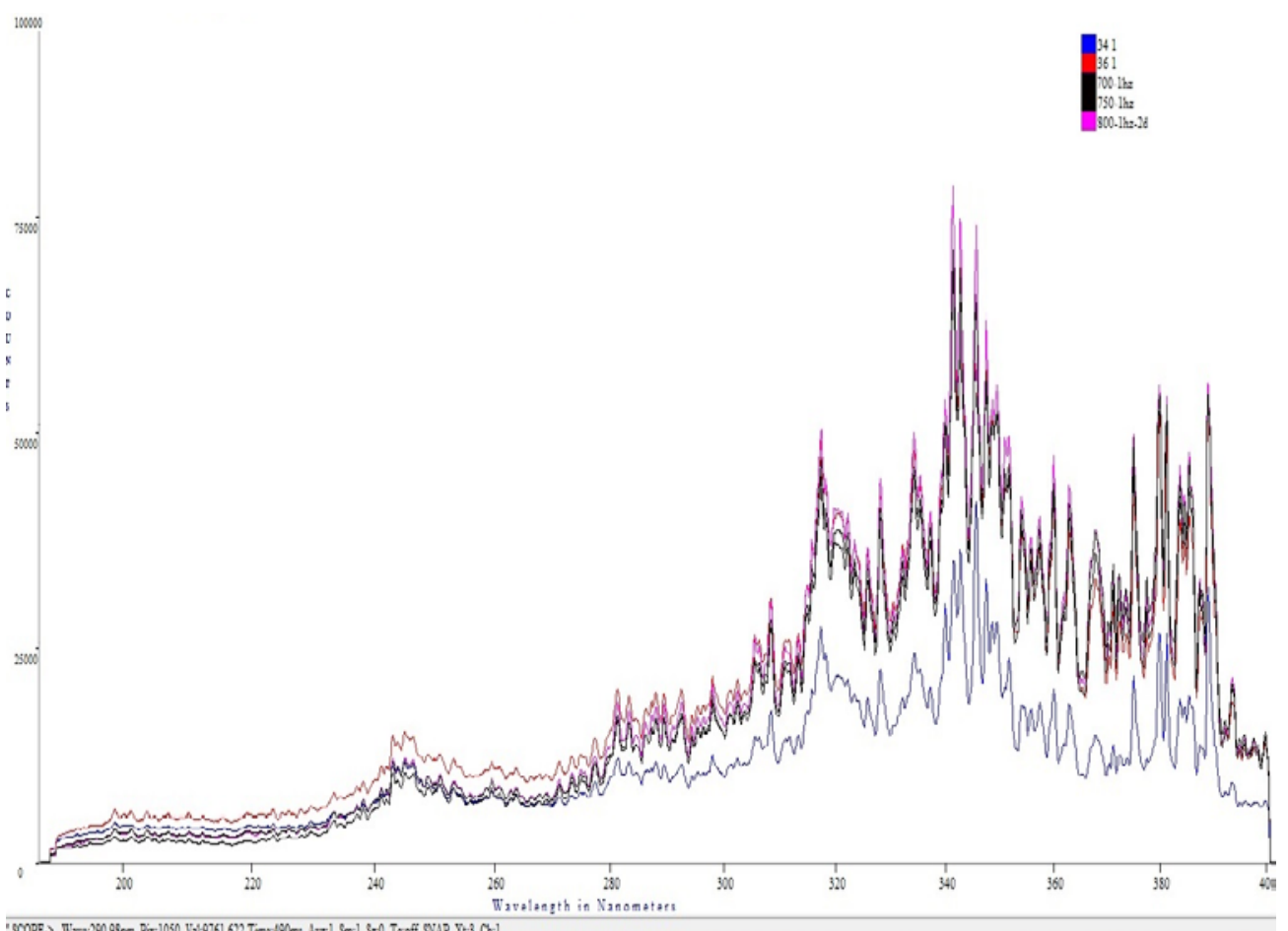

Fig.7: The emission spectra of laser induced holmium plasma with different laser energies and $(1 \mathrm{~Hz})$ repetition rate.

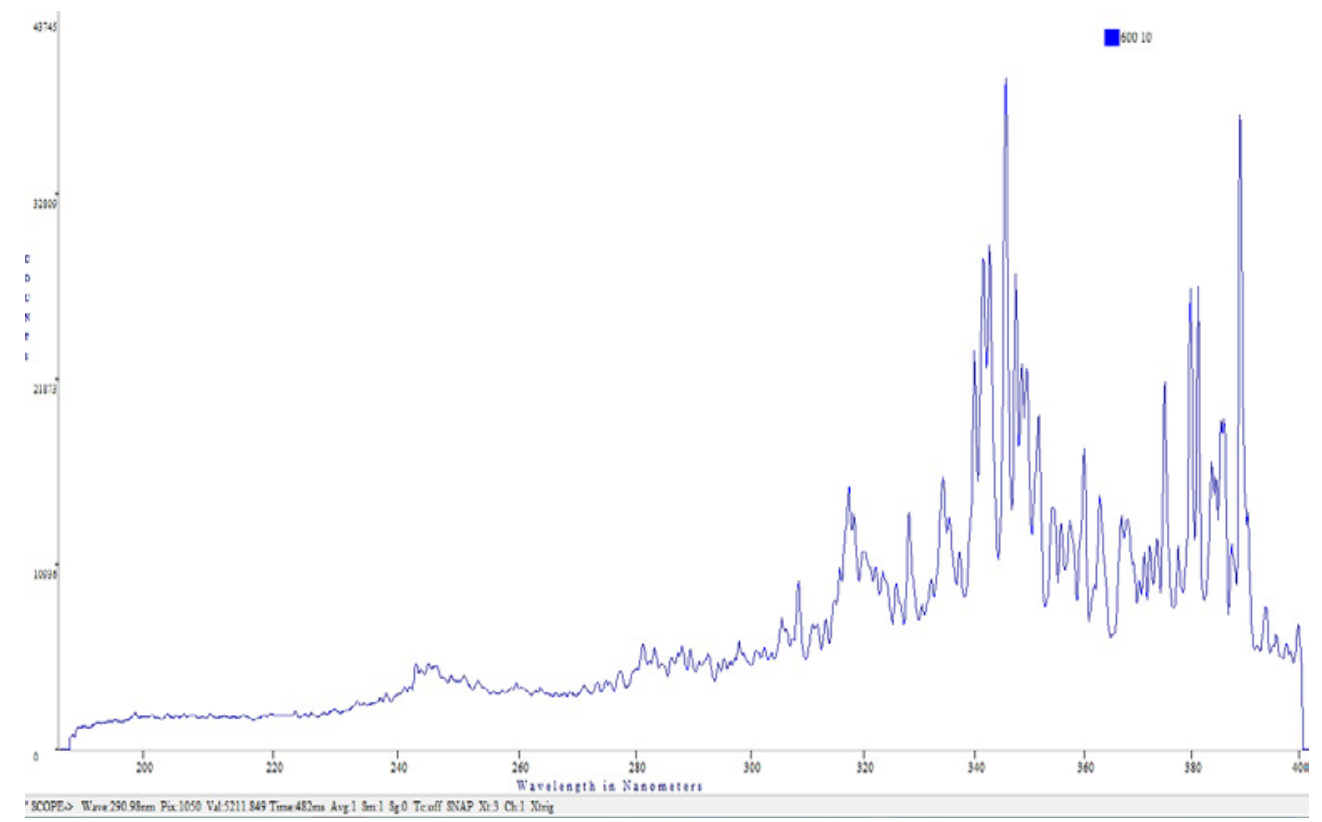

Fig.8: Emission spectra of laser energy $600 \mathrm{~mJ}$ with $10 \mathrm{~Hz}$ repetition rate. 


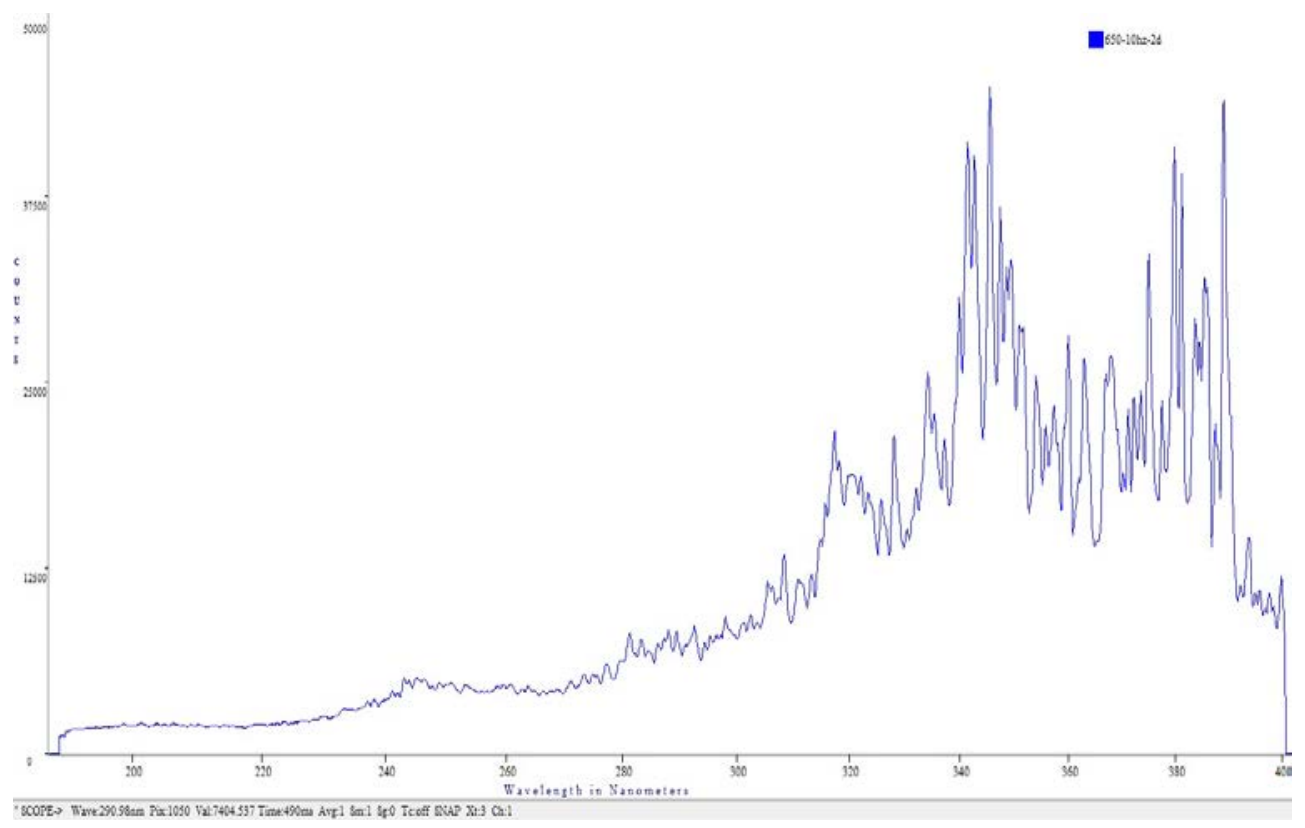

Fig.9: Emission spectra of laser energy $650 \mathrm{~mJ}$ with $(10 \mathrm{~Hz})$ repetition rate.

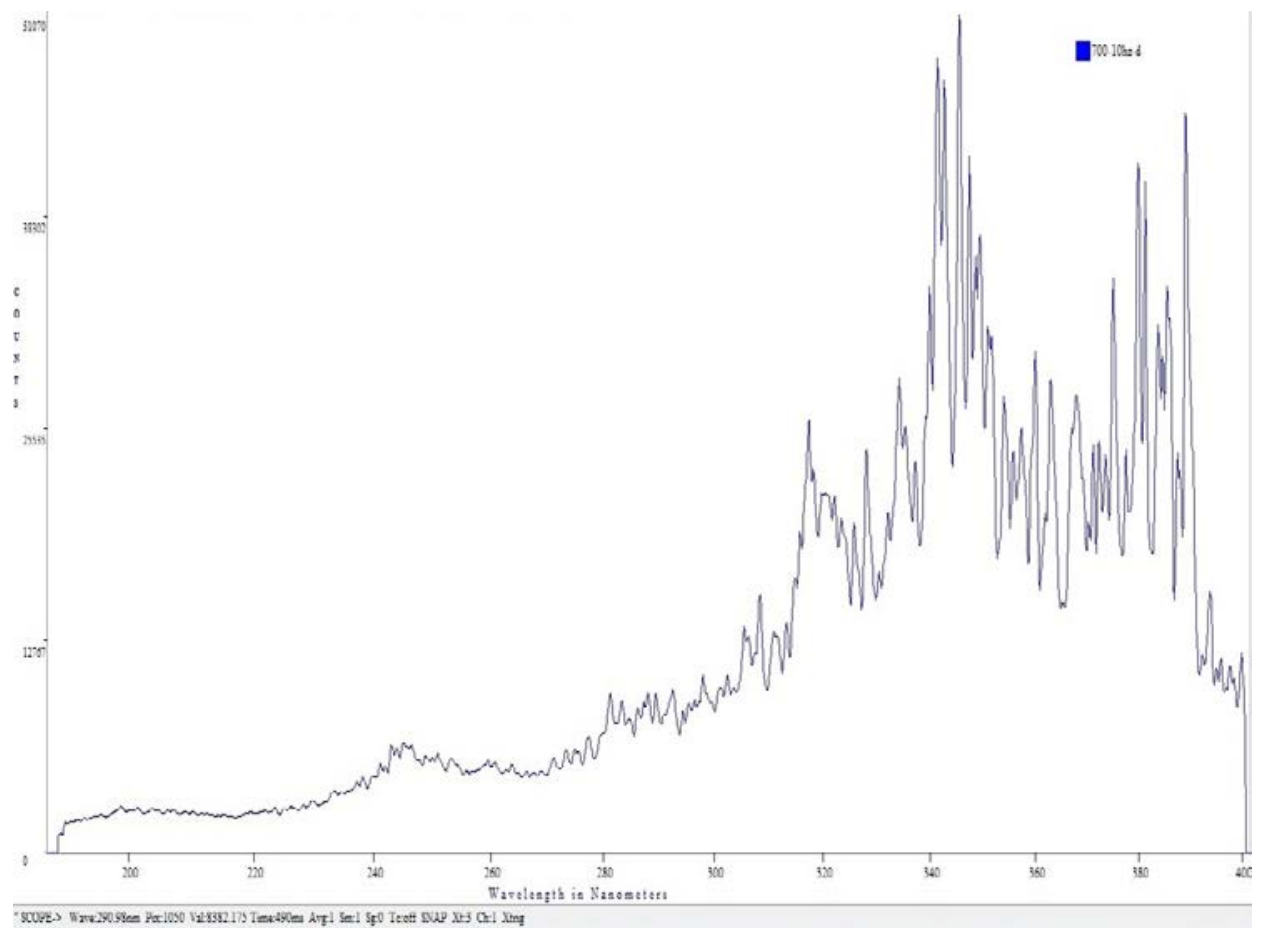

Fig.10: Emission spectra of laser energy $700 \mathrm{~mJ}$ with $10 \mathrm{~Hz}$ repetition rate.

The maximum intensity was (65000 at $\lambda=345.53 \mathrm{~nm}$ ) had been appeared when the laser pulse energy $750 \mathrm{~mJ}$, as shown in Fig.11.

The results of Fig.12 explain that, when the laser pulse energy increased to $800 \mathrm{~mJ}$, the maximum intensity of the holmium line emission spectra was $(77000$ at $\lambda=341.64 \mathrm{~nm})$. 


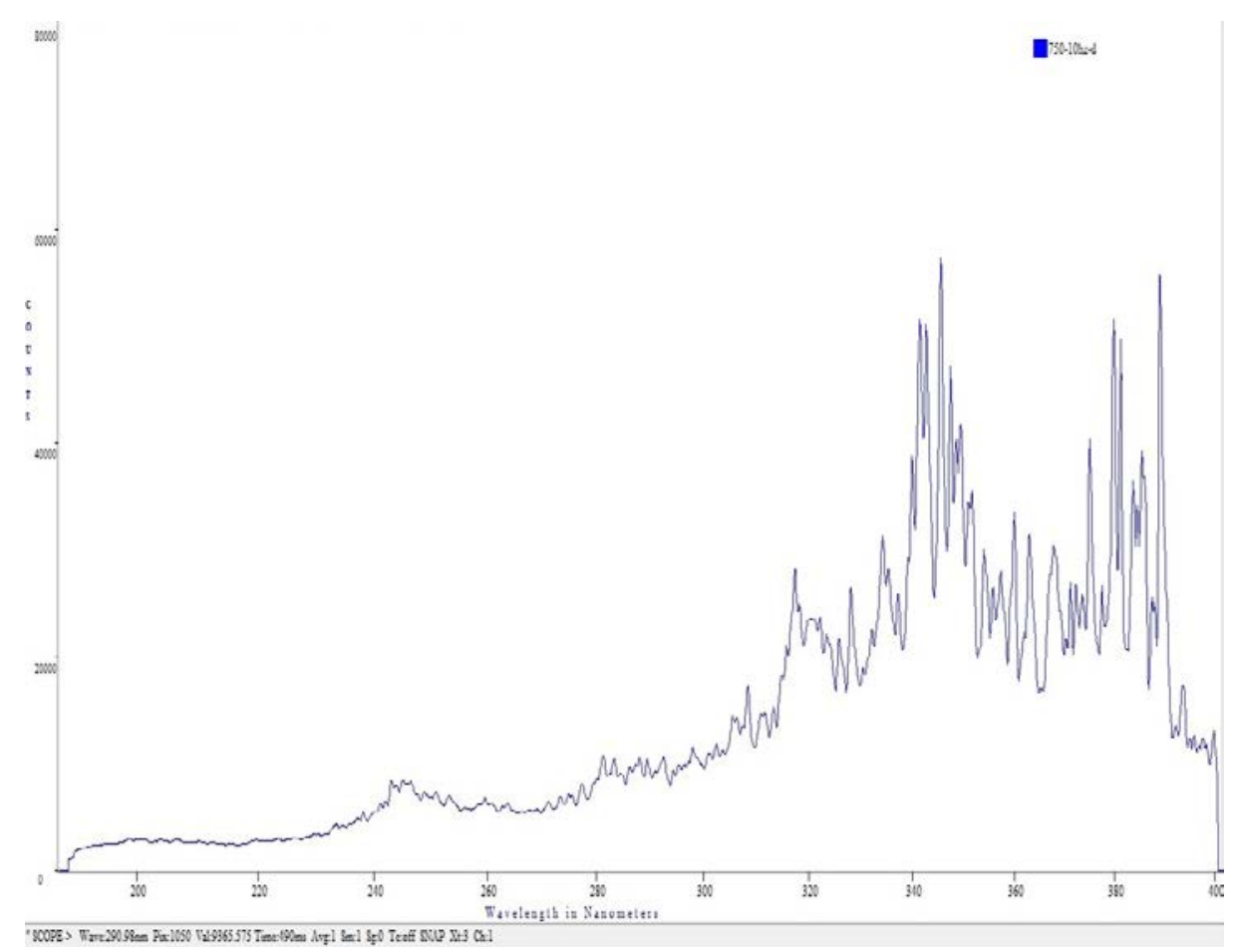

Fig.11: Emission spectra of laser energy $750 \mathrm{~mJ}$ with $10 \mathrm{~Hz}$ repetition rate.

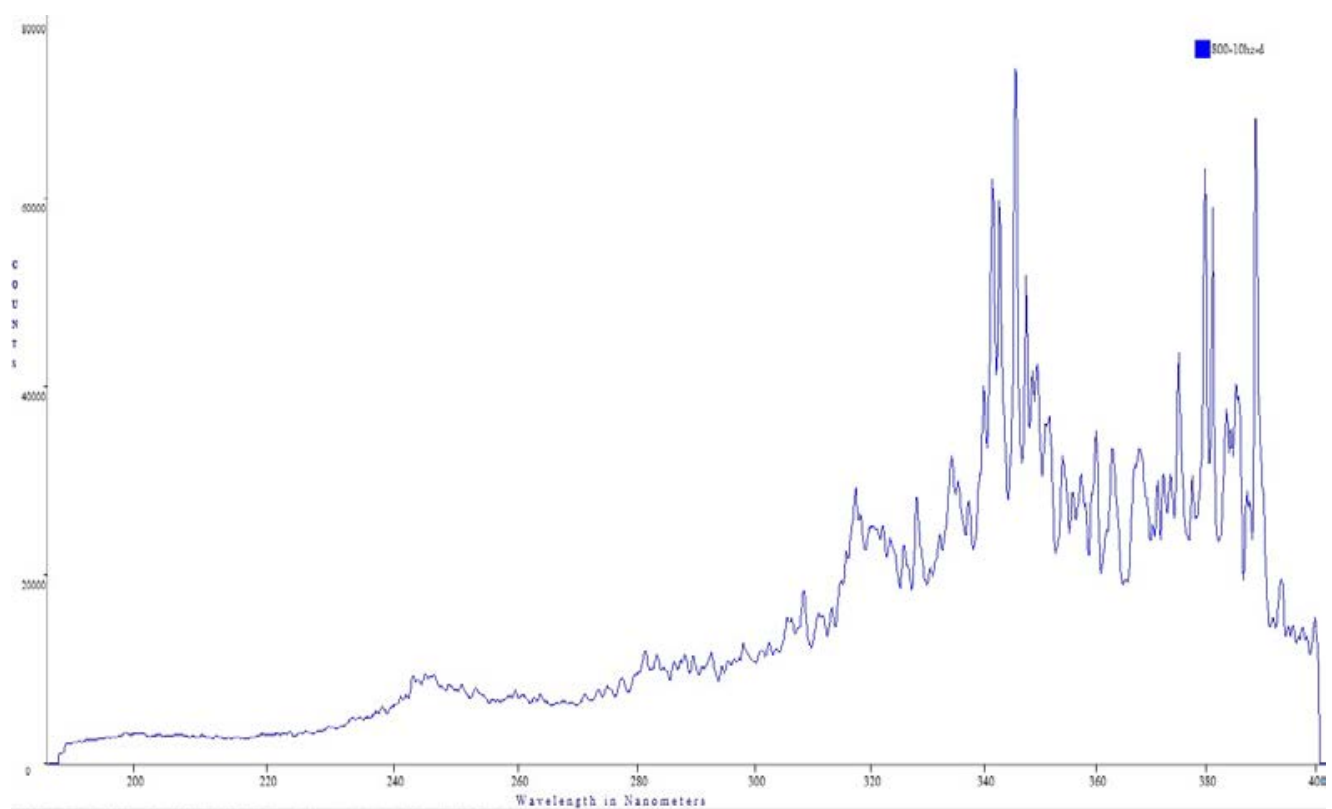

Fig.12: Emission spectra of laser energy $800 \mathrm{~mJ}$ with $10 \mathrm{~Hz}$ repetition rate.

Fig.13 shows all the results for different laser energies at $10 \mathrm{~Hz}$ as a repetition rate. The laser parameters used in the first and second group with wavelength and intensity, are listed in Table 1 and 2. 


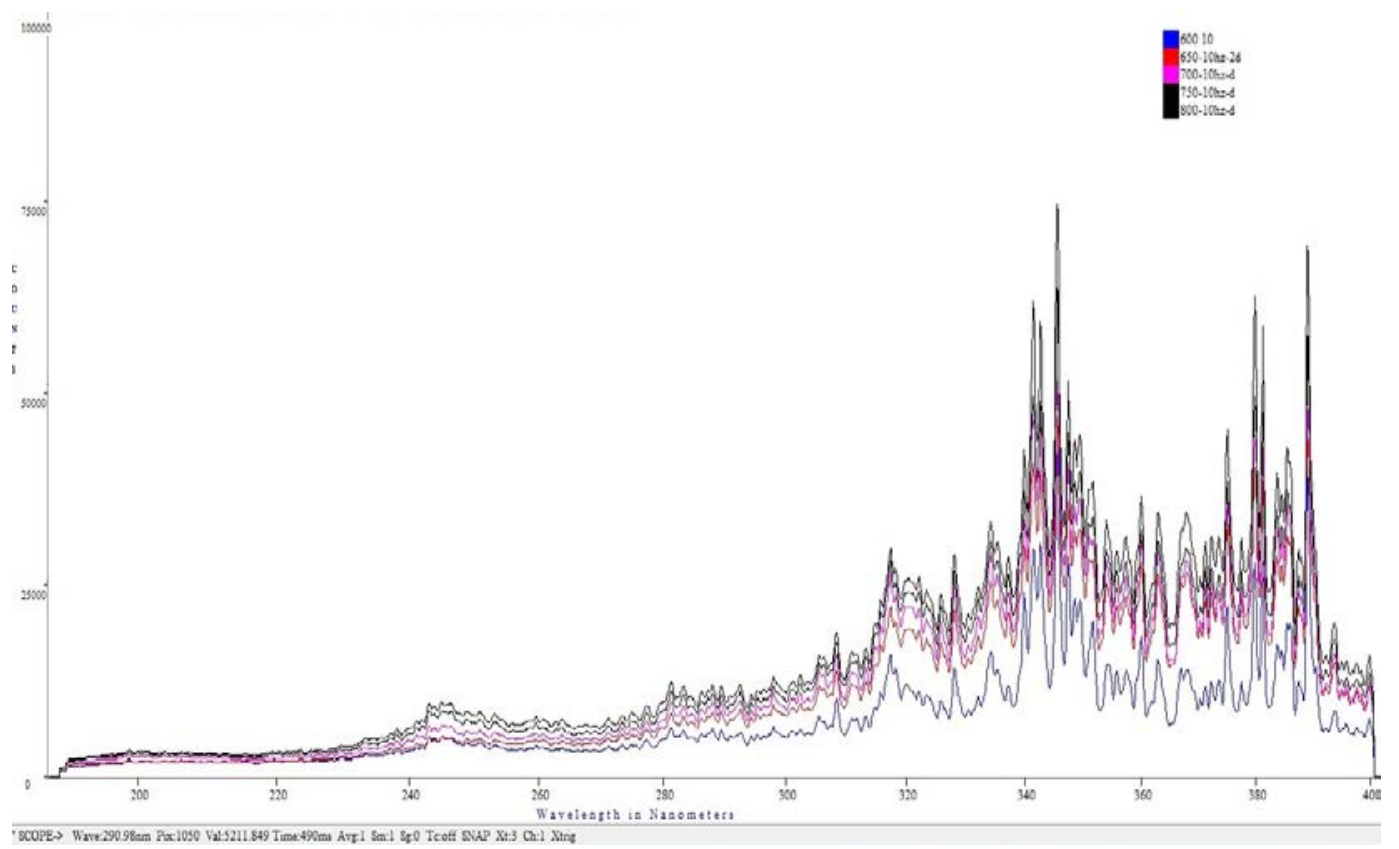

Fig.13: The emission spectra of laser induced holmium plasma with different laser energies and $(10 \mathrm{~Hz})$ repetition rate.

Table1: The laser parameters used in the first group at $(1 \mathrm{~Hz})$ repetition rate with wavelength and intensity.

\begin{tabular}{|c|c|c|}
\hline Energy(mJ) & Wavelength(nm) & intensity(counts) \\
\hline $\mathbf{6 0 0}$ & 345.75 & 40000 \\
\hline $\mathbf{6 5 0}$ & 345.75 & 59000 \\
\hline $\mathbf{7 0 0}$ & 341.43 & 65535 \\
\hline $\mathbf{7 5 0}$ & 341.54 & 70000 \\
\hline $\mathbf{8 0 0}$ & 341.54 & 80000 \\
\hline
\end{tabular}

Table 2: The laser parameters used in the second group at $(10 \mathrm{~Hz})$ repetition rate with wavelength and intensity.

\begin{tabular}{|c|c|c|}
\hline Energy(m) & Wavelength(nm) & Intensity(counts) \\
\hline $\mathbf{6 0 0}$ & 345.75 & 40000 \\
\hline $\mathbf{6 5 0}$ & 345.53 & 45000 \\
\hline $\mathbf{7 0 0}$ & 345.53 & 51070 \\
\hline $\mathbf{7 5 0}$ & 345.53 & 65000 \\
\hline $\mathbf{8 0 0}$ & 341.64 & 77000 \\
\hline
\end{tabular}

From the previous results, it can be seen that the intensities of the emission lines increase with increasing of the laser pulse energy and repetition rate [18]. The increasing of the pulse energy means the increase of its absorption by the plasma leading to more ablation from the target and finally increasing of the emission line intensity [19].
Fig.14 represents the emission spectra for Ho III (Tb like) ion. The second ionization stage of (Ho) theoretically computed using Cowan code covering wavelength region between 320 to $400 \mathrm{~nm}$. From computational method it has been illustrated that the emission spectra when $\lambda=345.64 \mathrm{~nm}$ is from transition (4f) - 5d. 


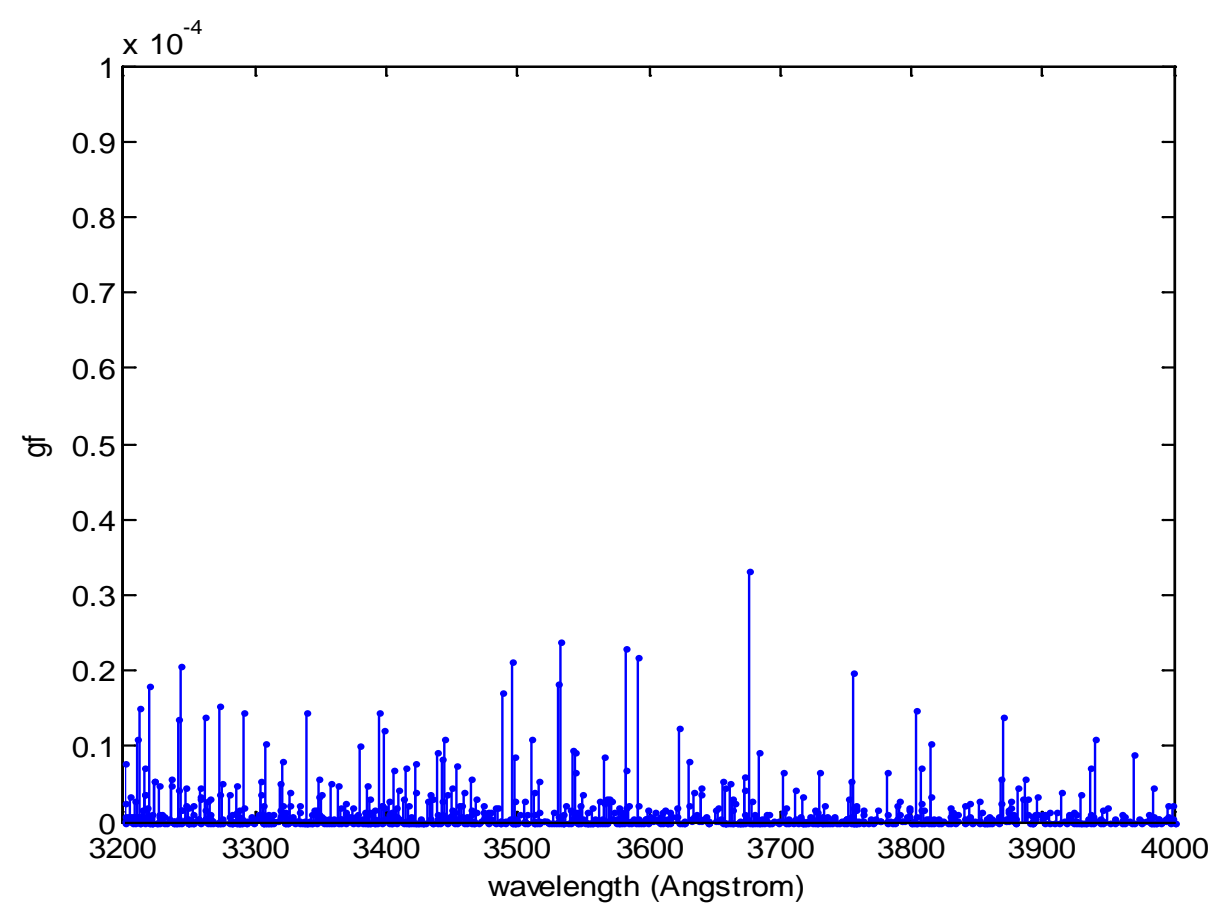

Fig.14: Emission spectrum of the second ionization of (Ho III).

\section{Conclusion}

The emission spectroscopy of the laser-induced plasma in air was investigated. The spectral line intensities of the laser induced plasma emission exhibited a strong dependence on pulsed laser energy and repetition rate

The increasing of the laser pulse energy to $800 \mathrm{~mJ}$ and $10 \mathrm{~Hz}$ repetition rate new line maximum emission intensity appeared at $\lambda=345.64 \mathrm{~nm}$. Theoretically using Cowan code have been assigned as transition from (4f) $5 d$.

From these results, it can be concluded that the emission intensity shows a marked dependence on the laser energy and repetition rate. Also, other different emissions can appear during this process according to different transition.

\section{References}

[1] N. S. Mazhir, A. N. Abdullah, F. A. Rauuf, H. A. Ali, I. H. AlAhmed, Baghdad Science J., 15, 1 (2018) 81-86.
[2] S. M. Mahmoad, Iraqi Journal of Physics (IJP), 16, 38 (2018) 83-98

[3] H. G. Jihad \& A. K. Aadim, Iraqi Journal of Physics, 16, 38 (2018) 1-9.

[4] A. K. Aadim, Iraqi Journal of Science, 56, 3B (2015) 2292-2296.

[5] H. H. Murbat \& A. H. Hamza, J. Mater Sci. Appl., 3 (2017) 1-7.

[6] G.M. Kompitsas, F. RoubaniKalantzopoulou, I. Bassiotis, A. Diamantopoulou, A. Giannoudakos, Proceedings of EARSeL-SIGWorkshop LIDAR, 1 (2000) 130-138. [7] R. d'Agostino, P. Favia, C. Oehr, R. M. Wertheimer, Plasma Processes and Polymers, 2, 1 (2005) 7-15.

[8] L. Fechner, "High-Resolution Experiments on Strong-Field Ionization of Atoms and Molecules: Test of Tunneling Theory, the Role of Doubly Excited States, and ChannelSelective Electron Spectra". Springer (2016).

[9] B. Cagnac and J. C. PebayPeyroula, "Modern Atomic PhysicsFundamental Principles", (Macmillan, London, 1975), Chap. 7, pp. 168-190. 
[10] M. Corsi, G. Cristoforetti, M. Hidalgo, S. Legnaioli, V. Palleschi, A. Salvetti, C.Vallebona, Applied Geochemistry, 21, 5 (2006) 748-755. [11] G. Başar, N. Al-Labady, B. Özdalgiç, F. Güzelçimen, A. Er, K.I. Öztürk, S. Kröger, The Astrophysical Journal Supplement Series, 228, 2 (2017) 1-6.

[12] N. Al-Labady, B. Özdalgiç, A. Er, F.Güzelçimen, K. I. Öztürk, S. Kröger, G. Başar, The Astrophysical Journal Supplement Series, 228, 2 (2017) 1-6.

[13] J. Gurell, M. G. Wahlgren, G. Nave, F. J. Wyart, Wavelengths, energy levels and hyperfine structure constants in Ho ii. Physica Scripta, 79, 3 (2009) 035306.
[14] G. Nave, Journal of the Optical Society of America B (JOSA), 20, 10 (2003) 2193-2202.

[15] R. D. Cowan, Journal of Optical Society of America, 58 (1968) 808818.

[16] R. D. Cowan, "The Theory of Atomic Structure and Spectra", California University Press (1981).

[17] R. D. Cowan, Phys. Rev., 163 (1967) 54-61.

[18] M. Kuzuya, H. Matsumoto, H.Takechi, O. Mikami, Journal of the Spectroscopical Society of Japan, 41, 5 (1992) 327-331.

[19] H. Legall, C. Schwanke, J. Bonse, J. Krüger, Applied Physics A, 125, 570 (2019) 1-8. 